\title{
CARACTERÍSTICAS DE DIABÉTICOS TIPO 2 ATENDIDOS EM CENTRO DE REFERÊNCIA DA CIDADE DE RECIFE/PE
}

\author{
CHARACTERISTICS OF DIABETICS TYPE 2 AT A REFERENCE CENTER OF \\ THE CITY OF RECIFE / PE \\ Joana Marcela Sales de Lucena' \\ Leone Severino do Nascimento ${ }^{2}$ \\ Denise Maria Martins Vancea ${ }^{3}$
}

\begin{abstract}
Resumo
O objetivo deste estudo foi comparar as variáveis antropométricas e metabólicas de indivíduos com DM2 praticantes de um programa de exercício físico supervisionado e não praticantes. Este é um estudo descritivo, exploratório com delineamento transversal. A amostra foi composta por dois grupos de idosos: o grupo praticante de exercícios físicos (GPEF) formado 09 mulheres e um homem e um grupo de idosos não praticantes de exercícios físicos (GNPEF) formado por 06 mulheres e 03 homens escolhidos aleatoriamente entre os idosos com diabetes tipo 2 atendidos no Centro de Saúde Senador José Ermírio de Moraes. Foram coletadas informações sociodemográficas, variáveis metabólicas (HbA1c e glicemia de jejum), prática de exercícios físicos, medidas de estatura, massa corporal, relação cintura/quadril (RCQ), circunferência de cintura (CC), sendo posteriormente calculado o IMC. Os dois grupos apresentaram valores elevados de CC e RCQ, sendo maior no GNPEF. A RCQ apresentou valores menores no GPEF quando comparada ao GNPEF. Quanto aos valores da HbA1c, o GNPEF apresentou níveis elevados, enquanto o GPEF encontrava-se dentro dos valores de referência. A prática de exercícios físicos aparentemente contribuiu para a diminuição da glicemia de jejum e manutenção dos parâmetros metabólicos dos diabéticos tipo 2 que praticavam exercícios físicos.
\end{abstract}

Palavras-chave: Diabetes mellitus. Exercício físico. Antropometria.

\begin{abstract}
The objective of this study was to compare the anthropometric and metabolic variables of individuals with DM2 who were practicing a supervised physical exercise program and not practicing them. This is a descriptive, exploratory study with a cross-sectional design. The sample consisted of two groups of elderly: the physical exercise group (GPEF) comprised 09 women and one man and a group of non-physical exercise elderly people (GNPEF) composed of 06 women and 03 men randomly chosen among the elderly with type 2 diabetes treated at the Senador José Ermírio de Moraes Health Center. Socio-demographic information, metabolic variables (HbAlc and fasting glycemia), physical exercise, height measures, body mass, waist / hip ratio), waist circumference, and BMI were subsequently calculated. The two groups presented high values of waist circumference and waist / hip ratio, being higher in GNPEF. The waist/hip ratio presented lower values in GPEF when compared to GNPEF. Regarding the HbA1c values, the GNPEF presented high levels, while the GPEF was within the reference values. The practice of physical exercises apparently contributed to the reduction of fasting blood glucose and maintenance of the metabolic parameters of type 2 diabetics who practiced physical exercises.
\end{abstract}

Keywords: Diabetes mellitus. Physical exercise. Anthropometry.

1. Professora Assistente da Universidade Federal do Tocantins - UFT. 2. Professor do Instituto Federal de Pernambuco - IFPE. 3. Professora Adjunta da Universidade Federal de Pernambuco - UPE.

Autor para correspondência: Joana Marcela Sales de Lucena. Rua: Av. Nossa Senhora de Fátima, sem número, Centro, Tocantinópolis/TO. 


\section{INTRODUÇÃO}

Estima-se que em 2030, o Brasil terá cerca de 11 milhões de pessoas com diabetes ${ }^{1}$. Segundo dados do Vigitel realizado em 2016, a prevalência de diabetes aumenta com a idade e cresceu 61,8\% na última década ${ }^{2}$. Considerada uma doença altamente incapacitante e uma das principais causas de mortalidade, insuficiência renal, amputação de membros inferiores, cegueira e doença cardiovascular $^{3}$. Os fatores que podem contribuir para o surgimento do diabetes são o aumento no IMC (índice de massa corporal) e o sedentarismo favorecido pela vida moderna (dieta inadequada e diminuição do nível de atividade física ${ }^{4}$.

O diabetes mellitus tipo 2 (DM2) é a forma da doença presente em 90\%-95\% dos casos. Entre as hipóteses sobre a causa do DM2 considera-se que há produção de insulina pelo pâncreas, porém as células musculares e adiposas não são capazes de reconhecê-la e o organismo não consegue metabolizar a glicose presente na corrente sanguínea, fenômeno conhecido como resistência insulínica ${ }^{5}$.

O principal objetivo no tratamento do diabetes é o controle glicêmico, indicado pela glicemia de jejum e pela $\mathrm{HbA1c}$ (hemoglobina glicada) cujos níveis adequados são $126 \mathrm{mg} / \mathrm{dl}$ e $\leq 7 \%$, respectivamente, acima dos quais aumentase o risco de doença macrovascular ${ }^{5}$. Tal controle glicêmico pode ser alcançado por meio de medicamentos, dieta adequada e exercícios físicos regulares. $\mathrm{O}$ exercício físico pode promover melhoras no perfil glicêmico $^{10}$ e na composição corporal do DM2 $2^{1,1,12,13}$.

As mudanças na aptidão física e na composição corporal ocorrem em decorrência da prática de exercícios físicos, e os diabéticos que praticam exercícios podem apresentar um IMC e um perfil glicêmico muito melhor quando comparados aos diabéticos que recebem apenas o tratamento tradicional (medicamentos e dieta), ou seja, não praticam exercícios físicos ${ }^{18}$. Portanto, o objetivo deste estudo foi comparar as variáveis antropométricas e metabólicas de indivíduos com DM2 praticantes de um programa de exercício físico supervisionado e não praticantes.

\section{MÉTODOS}

Trata-se de um estudo descritivo, exploratório com delineamento transversal. A amostra foi composta por dois grupos de idosos: o grupo praticante de exercícios físicos (GPEF) formado 09 mulheres e um homem, frequentadores da Academia da Cidade do Centro de Saúde Senador José Ermírio de Moraes; e um grupo de idosos não praticantes de exercícios físicos (GNPEF) formado por 06 mulheres e 03 homens escolhidos aleatoriamente entre os idosos com diabetes tipo 2 atendidos no 
Centro de Saúde Senador José Ermírio de Moraes que não participavam da Academia da Cidade.

Para participar deste estudo, todos os participantes assinaram um Termo de Consentimento Livre e Esclarecido. O projeto foi submetido e aprovado pelo Comitê de Ética em Pesquisa da Universidade Federal de Pernambuco (Protocolo nº523/11).

A coleta de dados foi realizada num período de 15 dias úteis, durante os quais foram coletados os dados de ambos os grupos paralelamente. Inicialmente foi realizada uma análise das fichas dos frequentadores da Academia da Cidade do Centro de Saúde Senador José Ermírio de Moraes, para composição do GPEF conforme os critérios de inclusão (idade a partir de 60 anos, e ser portador de diabetes tipo 2, independente do uso de insulina) e posterior verificação da frequência dos indivíduos selecionados no programa de exercícios.

Para o GNPEF foram selecionados os indivíduos que aguardavam a consulta médica na sala de espera do Centro de Saúde Senador José Ermírio de Moraes que se encaixaram nos critérios de inclusão (idade a partir de 60 anos e ser portador de diabetes tipo 2, independente do uso de insulina e não estar praticando nenhum exercício físico regularmente há pelo menos três meses). Para ambos os grupos foram utilizados os seguintes critérios de exclusão: idade abaixo de 60 anos, não ser portador de diabetes tipo 2 e não ser atendido pelo serviço de endocrinologia do Centro de Saúde Senador José Ermírio de Moraes.

Os dados referentes às informações sócio-demográficas, variáveis metabólicas (HbA1c e glicemia de jejum) e prática de exercícios físicos, foram coletados nos prontuários e exames encontrados no serviço de Arquivo do Centro de Saúde Senador José Ermírio de Moraes. As medidas das variáveis antropométricas em ambos os grupos foram IMC, relação cintura/quadril (RCQ) e circunferência de cintura (CC), coletadas pelo pesquisador. $\mathrm{O}$ IMC foi calculado por meio da massa corporal dividida pela estatura ao quadrado: massa corporal $(\mathrm{Kg}) /$ estatura $\left(\mathrm{m}^{2}\right)^{19}$.

A estatura foi medida pregando-se uma fita métrica na parede (após a marca de $1 \mathrm{~m}$ ), com o indivíduo descalço, de costas para a parede, olhando para frente, e ao inspirar profundamente e prender a respiração, foi registrada a altura em centímetros. massa corporal e o percentual de gordura foram mensurados utilizando-se uma balança digital, marca G-Tech, com o indivíduo sem sapatos, orientado a subir na balança enquanto eram registrados o peso e o percentual de gordura, este último através de bioimpedância.

Para medidas da RCQ utilizou-se uma trena antropométrica, marca Sanny, a 
medida de cintura foi realizada considerando-se a menor circunferência acima do umbigo e a medida do quadril foi realizada considerando-se a maior circunferência ao redor das nádegas. A RCQ foi calculada dividindo-se a circunferência da cintura pela circunferência do quadril: RCQ = circunferência da cintura/circunferência do quadril $^{19}$.

Para análise estatística foi realizada uma análise descritiva dos resultados contemplando média e desvio padrão. Para comparação entre os grupos foi utilizado o teste $t$ de Student para grupos independentes com auxílio do programa estatístico SPSS versão 10.0.

\section{RESULTADOS E DISCUSSÃO}

Para composição da amostra do GPEF foram selecionados inicialmente 19 sujeitos que se encaixaram nos critérios de inclusão, porém 09 não permaneceram na amostra final, por não comparecerem ao programa de exercícios físicos ou pelos dados insuficientes nos prontuários. Sendo assim, o GPEF foi composto por 10 sujeitos com idade média de $68,60 \pm 5,91$, tempo de diagnóstico de diabetes mellitus 10,1 \pm 6,40 e apenas um utilizava insulina (tabela $1)$.

Dos sujeitos escolhidos aleatoriamente para composição do GNPEF, 17 participaram da coleta de dados, dos quais 08 foram excluídos da amostra por dados insuficientes nos prontuários, finalizando o GNPEF com 09 sujeitos com idade média de 69,0 $\pm 7,28$ anos, tempo de diagnóstico de diabetes mellitus $11,56 \pm 9,60$ anos e, dos quais cinco faziam uso de insulina (tabela 1). Neste estudo, as variáveis antropométricas (peso corporal, RCQ e GJ) do GNPEF apresentaram-se mais elevadas do que no GPEF.

Tabela 1: Caracterização da amostra de diabéticos atendidos no Centro de Referência Senador José Ermírio de Morais em Recife/PE.

\begin{tabular}{lcc}
\hline Variável & GPEF & GNPEF \\
\hline Sexo & $9(\mathrm{~F}) / 1(\mathrm{M})$ & $6(\mathrm{~F}) / 3(\mathrm{M})$ \\
Idade & $68,60^{*} \pm 5,91^{* *}$ & $69,0^{*} \pm 7,28^{* *}$ \\
TMDD & $10,1^{*} \pm 6,40^{* *}$ & $11,56^{*} \pm 9,60^{* *}$ \\
$\begin{array}{l}\text { Uso de } \\
\text { Insulina }\end{array}$ & 9 (não) / 1 (sim) & 4 (não) $/ 5($ sim) \\
\hline
\end{tabular}

Legendas: GNPEF: Grupo não praticante de exercícios físicos; GPEF: grupo praticante de exercícios físicos; TMDD: tempo médio de diagnóstico de diabetes. *valores da média; * * desvio padrão.

Os dois grupos apresentaram valores elevados de CC e RCQ, ou seja, ambos possuem fatores de risco para doenças metabólicas e cardiovasculares ${ }^{16,20}$, porém o GNPEF apresentou valores maiores quando comparado ao GPEF (tabela 2). Este dado está de acordo com os achados de outros estudos, nos quais eram encontrados valores elevados de circunferência abdominal e circunferência de cintura em idosos com DM 2 e associação entre vários fatores de risco para doença cardiovascular ${ }^{21}$, como o próprio 
diabetes, a hipertensão arterial e dislipidemias, assim como um nível de atividade física inadequado ${ }^{22,23}$.

Existe uma relação positiva entre CC, obesidade central e doença cardiovascular, sendo possível ocorrer uma melhora decorrente do exercício físico sobre a $\mathrm{CC}^{11}$. Assim, ao compararmos os dois grupos, provavelmente a participação num programa de exercícios supervisionado evitou um aumento maior e mais significativo da CC do GPEF, dados conhecidos os efeitos do exercício físico nesta variável.

A RCQ apresentou valores menores no GPEF quando comparada ao GNPEF, porém o risco para hipertensão, hiperlipidemia e doença arterial coronariana (DAC) ainda foi considerado muito alto para ambos os grupos ${ }^{19}$. Tal resultado pode estar relacionado à idade da população estudada, pois em um estudo realizado com idosos aqueles com DM2 apresentaram valores acima do recomendado para RCQ - $84 \%$ apresentaram valores elevados numa população de 100 idosos, os quais também apresentaram nível de atividade física inadequado ${ }^{22}$.

Outro estudo demonstrou que um aumento na RCQ induziria a um aumento na massa corporal total (MCT) e na glicemia dos indivíduos. Para cada aumento de 0,01 da RCQ haveria um aumento de
$1 \mathrm{Kg}$ na MCT e para cada 0,1 da RCQ haveria aumento de $15 \mathrm{mg} / \mathrm{dL}$ na glicemia, ou seja, à medida que aumenta a massa corporal total, indicada pela RCQ, aumentam os níveis glicêmicos ${ }^{24}$. Um aumento da CC e da RCQ pode estar também associado ao aumento na GJ e maiores níveis glicêmicos, de triglicérides e colesterol assim como menor atividade física, conforme os achados de outros estudos sobre a associação existente entre perfil metabólico irregular e adiposidade central, tanto em homens como mulheres (gordura visceral) $^{25,26}$.

Quanto aos valores da HbAlc, o GNPEF apresentou níveis elevados, enquanto o GPEF encontrava-se dentro dos valores de referência $(7 \%)^{27}$. Ao comparar os grupos, apesar de não ser estatisticamente significativa, ocorreu uma diferença interessante entre os valores (tabela 2). A HbA1c é um indicador da saúde metabólica do indivíduo com DM 2 e seu controle é essencial na prevenção de complicações micro e macrovasculares. A prática de exercícios regulares como caminhada, exercícios de força ou a combinação dessas duas modalidades tem demonstrado mudanças no perfil antropométrico e glicêmico do DM2 $2^{11,12,1,2,28,29}$. Porém, o efeito do programa de exercícios sobre a RCQ, CC, GJ e HbA1c parece estar relacionado principalmente a dois fatores: o tempo de participação e a 
frequência semanal ao programa de exercícios físicos.

Neste estudo foi encontrada uma diferença estatisticamente significativa na glicemia de jejum dos grupos e valores adequado de HbA1c no GPEF. Os estudos que aplicaram algum programa de exercício físico $^{12,13,30}$ encontraram resultados nas glicemias após 10 semanas, 13 semanas e 16 semanas de exercícios físicos, respectivamente. De fato, o GPEF teve um tempo de prática variando entre 03 meses a 01 ano, concordando com os achados dos outros estudos e demonstrando uma manutenção de valores metabólicos aceitáveis em longo prazo.

Os indivíduos do GPEF possuíram uma frequência de menos de $30 \%$ num período de 03 meses antes da coleta destes dados, o que corresponde a uma frequência de menos de 03 vezes por semana nesse período. A maioria dos estudos encontram efeitos sobre as variáveis metabólicas após programas de exercícios com frequência semanal de 3 a 4 vezes por semana, mas não encontram efeitos significativos sobre as variáveis antropométricas ${ }^{12,13,30}$, sendo tais efeitos encontrados quando a frequência do exercício chega a 05 vezes por semana ${ }^{11}$. Os resultados deste estudo indicam que efeitos estatisticamente significativos sobre as variáveis RCQ e CC poderiam ser encontrados se a frequência no programa de exercícios físicos fosse maior.
Tabela 2: Variáveis Antropométricas e Metabólicas (GPEF e GNPEF) de diabéticos atendidos no Centro de Referência Senador José Ermírio de Morais em Recife/PE.

\begin{tabular}{lccc}
\hline Variável & $\begin{array}{c}\text { GPEF } \\
(\text { média } \pm \text { DP) }\end{array}$ & $\begin{array}{c}\text { GNPEF } \\
(\text { média } \pm \text { DP) }\end{array}$ & p \\
\hline RCQ & $0,91 \pm 0,054 \dagger$ & $0,95 \pm 0,034 \dagger$ & 0,3 \\
$\mathbf{C C}$ & $96,45 \pm 9,176$ & $103,94^{*} \pm 8,21$ & 0,8 \\
Massa & & & \\
Corporal & $72,02 \pm 13,90$ & $83,61 \pm 17,21$ & 0,5 \\
$\mathbf{( K g )}$ & & & \\
HbA1c & $6,95 \pm 1,948$ & $8,07 \pm 2,12$ & 0,2 \\
GJ $\dagger$ & $131,60 \pm 23,38 \dagger$ & $150,44 \pm 82,19 \dagger$ & $0,01 \dagger$ \\
\hline
\end{tabular}

Legendas: $\dagger=$ resultado estatisticamente significativo. $\mathrm{p} \leq$ 0,05. DP: desvio-padrão, RCQ: relação cintura/quadril, CC: circunferência de cintura, HbA1c: hemoglobina glicada, GJ: glicemia de jejum;

\section{CONCLUSÃO}

Para esta amostra, os dados indicam que a prática de exercícios físicos levou à diminuição da glicemia de jejum assim como impediu que os parâmetros metabólicos dos diabéticos tipo 2 que praticavam exercícios físicos ultrapassassem os níveis recomendados quando comparados com o grupo que não praticava. Quanto às variáveis antropométricas, não foram encontradas diferenças significativas entre os grupos, provavelmente devido à frequência inadequada ao programa de exercícios, ou seja, a maioria não apresentou $75 \%$ de freqüência no programa.

São necessários mais estudos que avaliem as variáveis metabólicas e antropométricas de diabéticos tipo 2, assim como variáveis referentes aos exercícios 
físicos (frequência, tempo, modalidade, intensidade).

\section{REFERÊNCIAS}

1. WILD, S., ROGLIC, G., GREEN, A., SICREE, R., KING, H. Global Prevalence of Diabetes: Estimates for the year 2000 and projections for 2030. Diabetes Care, v. 27, n. 5, p. 1047 - 1053, 2004 .

2. BRASIL. Ministério da Saúde. Secretaria de Vigilância em Saúde. Departamento de Vigilância de Doenças e Agravos não Transmissíveis e Promoção da Saúde. Vigitel Brasil 2016: vigilância de fatores de risco e proteção para doenças crônicas por inquérito telefônico: estimativas sobre frequência e distribuição sociodemográfica de fatores de risco e proteção para doenças crônicas nas capitais dos 26 estados brasileiros e no Distrito Federal em 2016 / Ministério da Saúde, Secretaria de Vigilância em Saúde, Departamento de Vigilância de Doenças e Agravos não Transmissíveis e Promoção da Saúde. - Brasília: Ministério da Saúde, 2017.

3. BRASIL. Ministério da Saúde. Cadernos de Atenção Básica. Normas e Manuais Técnicos. Secretaria de Atenção à Saúde. Departamento de Atenção Básica. Brasília, n. 16, Série A, p. 09, 2006.

4. SARTORELLI D. S.; FRANCO L. J. Tendências do diabetes mellitus no Brasil: o papel da transição nutricional. Cadernos de Saúde Pública, Rio de Janeiro, n. 19, Sup. 1, p. 29-36, 2003.

5. SOCIEDADE BRASILEIRA DE DIABETES. Diretrizes da Sociedade Brasileira de diabetes 2009. $3^{\text {a }}$ edição, Itapevi, SP: AC Farmacêutica, 2009. Disponível em http://www.diabetes.org.br.
6. ALMEIDA, A. A .L. et al. Perfil Epidemiológico do Diabetes Mellitus AutoReferido em Uma Zona Urbana de Juiz de Fora, Minas Gerais. Arquivos Brasileiros de Endocrinologia e Metabologia, v. 43, n. 3, Junho, 1999.

7. COTTA, R. M. M. et al. Perfil sociossanitário e estilo de vida de hipertensos e/ou diabéticos usuários do Programa Saúde da Família. Revista Ciência e Saúde Coletiva, v. 14, n. 4, p. 1261-1260, 2009

8. FRANCISCO, P. M. S .B. et al. Diabetes auto-referido em idosos: prevalência, fatores associados e práticas de controle. Cadernos de Saúde Pública, Rio de Janeiro, v. 26, n. 1, p. 175-184, Janeiro, 2010 .

9. ROSA, R. S., SCHIMDT, M. I. Diabetes mellitus: magnitude das hospitalizações na rede pública do Brasil, 1999-2001. Epidemiologia e Serviços de Saúde, v.17, n.2, Brasília Junho, 2008.

10. PASSOS, A.P. et al. Diabetes mellitus tipo 2 e exercício físico aeróbico. Revista Diabetes Clínica, v. 6, n. 5, p. 375380, 2002.

11. VANCEA, D. M. M. et al. Efeito da freqüência do exercício físico no controle glicêmico e composição corporal de diabéticos tipo 2. Arquivos Brasileiros de Cardiologia, v. 1, n. 92, p. 23-30, 2009.

12. MONTEIRO, L. Z. et al. Redução da pressão arterial, do IMC e da glicose após treinamento aeróbico em idosas com diabete tipo 2. Arquivos Brasileiros de Cardiologia, v. 95 , n. 5 , p. 563-570, 2010.

13. SILVA, C. A., LIMA, W. C. Efeito Benéfico do Exercício Físico no Controle Metabólico do Diabetes Mellitus Tipo 2 à Curto Prazo. Arquivos Brasileiros de Endocrinologia e Metabologia, v. 46, n. 5, P. 550-556, Outubro, 2002. 
14. CHIAPPA, G.R.S. et al. Investigação da aptidão física de pacientes diabéticos não - insulinodependentes.Revista Kinesis, Santa Maria, n. 26, p. 37-50, 2002.

15. BRASIL. Ministério da Saúde. Inquérito domiciliar sobre comportamentos de risco e morbidade referida de doenças e agravos não transmissíveis: Brasil, 15 capitais e Distrito Federal, 2002-2003. p. 69, Rio de Janeiro: INCA, 2004. Disponível em http://www.inca.gov.br.

16. ALVES, L. R.; COUTINHO, V.; SANTOS, L. C. Indicadores antropométricos associados ao risco de doença cardiovascular. Arquivos Sanny Pesquisa e saúde, v. 1, n. 1, p. 1-7, 2008.

17. CASTRO, S. H., MATOS H. J., GOMES, M. B. Parâmetros Antropométricos e Síndrome Metabólica em Diabetes Tipo 2. Arquivos Brasileiros de Endocrinologia e Metabologia v. 50, n. 3, p. 450-455, Junho, 2006.

18. FRANCHI, K.M.B. et al. Comparação antropométrica e do perfil glicêmico de idosos diabéticos praticantes de atividade física regular e não praticantes. Revista Brasileira de Geriatria e Gerontologia, v. 13, n. 1, p. 73-81, Rio de Janeiro, 2010.

19. AMERICAN COLLEGE OF SPORTS MEDICINE. Manual do ACSM para avaliação da aptidão física relacionada à saúde. p. 43-51, Guanabara Koogan, 2008.

20. CAMBRI, L.T., GEVAERD, M.S. Indicadores antropométricos e parâmetros bioquímicos em diabéticos tipo 2. Motriz, Rio Claro, v.12, n.3, p.293 - 300, set./dez, 2006.

21. PICON, P.X. et al. Medida da Cintura e Razão Cintura/Quadril e Identificação de Situações de Risco
Cardiovascular: Estudo Multicêntrico em Pacientes Com Diabetes Melito tipo 2. Arq

22. Bras Endocrinol Metab, v. 3, n. 51, pag. 443-449, 2007.

23. SILVA, R.C.P., SIMÕES, M.J.S., LEITE, A.A. Fatores de risco para doenças cardiovasculares em idosos com diabetes mellitus tipo 2. Rev. Ciênc. Farm. Básica Apl, v. 28, n.1, p.113-121, 2007.

24. DEFAY, R., DELCOURT1, C., RANVIER, M., LACROUX1, A., PAPOZ1 L. AND POLA STUDY GROUP. Relationships between physical activity, obesity and diabetes mellitus in a French elderly population: the POLA study. International Journal of Obesity, v. 25, p. 512-518, 2001.

25. FIANI, C.R.V., MONTEIRO, L.Z., FOSS, M.C. Associação da Relação Cintura/Quadril com Glicemia e Massa Corporal em Diabéticos Tipo 2. Revista EPeQ/Fafibe, 1a. Ed., v. 1, pag. 29-35, 2010 .

26. VASQUES, A.C.J. et al. Influência do Excesso de Peso Corporal e da Adiposidade Central na Glicemia e no Perfil Lipídico de Pacientes Portadores de Diabetes Mellitus Tipo 2. Arq Bras Endocrinol Metab, v.51, n. 9, pag. 15161521, 2007.

27. YATES, T. et al. Levels of physical activity and relationship with markers of diabetes and cardiovascular disease risk in 5474 white European and South Asian adults screened for type 2 diabetes. Rev. Prev. Med., v.51, n. 3-4, p. 290, 2010.

28. SOCIEDADE BRASILEIRA DE DIABETES. Atualização sobre hemoglobina glicada (A1c) para avaliação do controle glicêmico e para o diagnóstico do diabetes:aspectos clínicos e laboratoriais, 2009. Retirado de http://www.diabetes.org.br/attachments/502 posicionamentos SBD 3 jan09.pdf 
29. CASTANEDA C. et al. A randomized controlled trial of resistance exercise training to improve glycemic control in older adults with type 2 diabetes. Diabetes Care, v. 25, n. 12, dez/2002.

30. MANJOO, P., JOSEPH, L., DASGUPTA, K. Abdominal adiposity and daily step counts as determinants of glycemic control in a cohort of patients with type 2 diabetes mellitus. Rev. Nutrition and Diabetes, p. 1- 6, 2012.

31. CAMBRI, L.T. et al. Efeito agudo e crônico do exercício físico no perfil glicêmico e lipídico em diabéticos tipo 2. Motriz, Rio Claro, v.13 n.4 p.238-248, out./dez. 2007. 